\title{
DRAWING BOARD
}

The drawing board can be made formany uses. One side can be used for technical drawing while the other side can be painted so that it can be wiped or washed clean after being used. If plywood or other manufactured board is not available thin seasoned timber can be glued and battened together, and sanded flat and smooth.

It is often better to make the board about the same size as the paper which will be used for technical drawing, for then clips can be used to hold the paper to the board instead of pins which always spoil the surface.

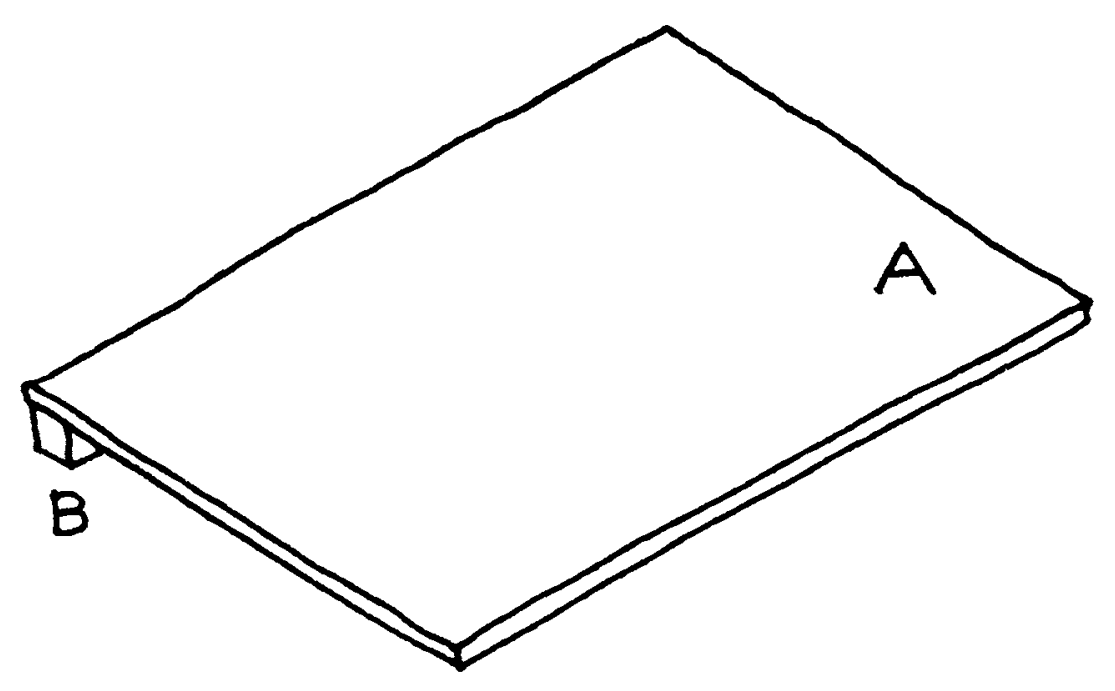

Construction - glue and nail

A Plywood $\frac{1}{4} "$ X $12 "$ x 18 "

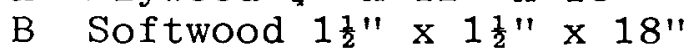

or Timber $\frac{1}{2} "$ x $12^{\prime \prime} \times 18 "$ 